\title{
Analyse d'une plate-forme d'innovation dans la filière karité au Mali
}

\author{
Amadou Sidibé ${ }^{1, *}$, Sietze Vellema ${ }^{2}$, Fadiala Dembelé ${ }^{1}$, Mamoudou Traoré ${ }^{1}$ et Thomas W. Kuyper ${ }^{3}$ \\ ${ }^{1}$ IPR/IFRA de Katibougou, BP 06, Koulikoro, Mali \\ 2 Knowledge, Technology and Innovation group, Wageningen University, Hollandseweg 1, NL-6706 KN, Wageningen, Pays-Bas \\ ${ }^{3}$ Department of Soil Quality, Wageningen University, Droevendaalsesteeg 4, NL-6708 PB, Wageningen, Pays-Bas
}

\begin{abstract}
Résumé - Lors de l'intervention d'une plate-forme d'innovation (groupe d'acteurs concernés) dans un domaine donné, nous avons tendance à attribuer les causes du changement aux actions de cette plate-forme. Cet article utilise le cas d'une plate-forme d'innovation dans le secteur du karité au Mali pour analyser comment les plates-formes d'innovation produisent les résultats escomptés. Le centrage des discussions et des actions de la plate-forme sur le thème de l'achat des amandes, et de la production et de la commercialisation du beurre de karité par les coopératives de femmes, a contribué à élargir le champ des opportunités qui s'offrent à celles-ci dans leur domaine. L'article met l'accent sur les processus qui découlent de la façon dont les activités de la plate-forme d'innovation entrent en résonnance avec les pratiques déjà en cours, dans un contexte en perpétuel changement. Il analyse le potentiel mais aussi les limites de la plate-forme dans les processus d'innovation, en confrontant les explications liées à la capacité de la plate-forme à entrer en synergie avec les efforts des acteurs de base, avec les explications qui tendent à attribuer les résultats du changement aux seules actions de la plate-forme.
\end{abstract}

\begin{abstract}
Analysis of an innovation platform in the shea nut value chain of Mali. Causal explanations of change after an Innovation Platform intervention in a given domain tend to attribute outcomes mainly to what the Innovation Platform did. This paper uses the case study of an Innovation Platform in the value chain of shea nut in Mali, to explore the actual processes that explain why and how innovation platforms lead to desired development outcomes. The articulation of innovation platform activities with the performance of a women's cooperative involved in sourcing shea kernels, and processing and trading shea butter, contributed to enlarging the space for women to navigate opportunities in the shea value chain. The paper emphasizes the underlying processes activated by the articulation between the Innovation Platform's activities and the already existing performance practices in a changing context. It confronts the explanatory power of these processes with assumptions that tend to attribute development outcomes only to what the Innovation Platform did, to unravel the potential and the limitation of Innovation Platforms in the innovation process.
\end{abstract}

\section{Introduction}

Les besoins de collaboration, de partage d'informations et de coordination des actions entre acteurs situés à différents échelons ont fait des plates-formes d'innovation (PI) un outil privilégié de développement, élargissant le cercle des acteurs au-delà des producteurs, vulgarisateurs et chercheurs depuis longtemps impliqués dans les programmes de développement agricole. L'approche PI est présumée susceptible d'impliquer un groupe d'acteurs plus large (Nederlof et al., 2011). Dans

\footnotetext{
$\bar{*}$ Auteur de correspondance $:$ sidibe.amadouy@gmail.com
}

cette approche, la stimulation et la facilitation des changements sociotechniques et institutionnels constituent le fondement de l'innovation. Une PI permet aux acteurs de communiquer et d'interagir de façon concertée (Nederlof et Pyburn, 2012). Cependant, les processus qui sous-tendent les activités des PI et qui expliquent pourquoi elles produisent certains résultats et pas d'autres sont moins bien connus (Nederlof et al., 2011).

La littérature sur l'innovation qui inspire l'approche PI met l'accent sur certains processus internes à la PI qui sont à l'origine des résultats obtenus. L'innovation émergerait de l'interaction et du processus d'apprentissage entre acteurs. Le postulat est que l'innovation nécessite un réseautage qui favorise l'apprentissage social et la gestion des dynamiques de 
pouvoir et de conflits (Leeuwis, 2004). Intermédiaires, facilitateurs et individus pouvant contribuer de façon décisive à l'innovation, ou encore « champions » soigneusement choisis (Hounkonnou et al., 2012 ; Klerkx et al., 2010), sont identifiés comme déterminants dans le changement. La coopération, le partenariat, le partage d'information et le co-apprentissage entre acteurs étatiques et non étatiques sont aussi habituellement identifiés comme déterminants (Spielman, 2005). L'objectif de cet article est de mettre l'accent sur le pouvoir explicatif qui découle de l'interaction entre la PI, les acteurs, les facteurs extérieurs, et les évènements externes qui se déroulent indépendamment de la plate-forme mais qui interagissent avec elle dans un contexte en perpétuelle évolution.

Une coopérative de femmes de la filière karité au Mali constitue ici le cadre d'intervention de la PI. Cette PI avait été créée afin de rechercher des solutions à certaines difficultés, techniques et institutionnelles rencontrées par ces femmes. L'article met l'accent sur les processus mis en œuvre par l'articulation entre les activités de la plate-forme et les pratiques des femmes telles que l'achat des amandes, la production et la commercialisation du beurre de karité.

\section{Le contexte de l'étude de cas}

Le karité (Vitellaria paradoxa C.F. Gaertn., syn. Butyrospermum paradoxum) est un arbre de la famille des Sapotaceae (Henry et al., 1983). Il pousse à l'état naturel dans les savanes d'Afrique de l'Ouest, où il est conservé dans les parcelles cultivées lors de leur défrichement. Les parcs à karité ne sont ainsi pas des formations naturelles, mais le résultat d'une sélection délibérée des génotypes présentant des traits désirables de fruits et de noix (APROMA, 1995). Une pulpe comestible enveloppe la noix. Elle est consommée en début de saison pluvieuse quand les réserves alimentaires des familles sont au plus bas. Cependant, le produit principal reste la noix, qui, après décorticage, fournit une amande dont est extrait le beurre de karité.

Le beurre de karité est un ingrédient essentiel du régime alimentaire et du commerce informel dans les zones où il est produit. Il est la principale matière grasse d'origine végétale utilisée dans la cuisine locale, mais est aussi utilisé comme matière première dans la fabrication de savon (Derks et Lusby, 2006). La part du Mali dans le commerce mondial du karité représente moins de $10 \%$ du total (Lusby et Derks, 2006). Le marché mondial concerne l'Afrique occidentale ainsi que des créneaux spécialisés en Europe et en Amérique du Nord. Après l'autorisation par l'Union européenne de l'utilisation de diverses matières grasses d'origine végétale comme équivalent du beurre de cacao dans l'industrie du chocolat, la demande du marché européen en amandes de karité a fortement augmenté et cela s'est ressenti sur les marchés locaux ouest-africains (Greig, 2006). La filière internationale du karité est, de façon générale, contrôlée essentiellement par des hommes et est dominée par quelques agents en situation de monopole (Fold et Reenberg, 1999).

Le Mali abrite environ 20 millions de pieds de karité. La production annuelle d'amandes est estimée à 100000 tonnes. Le karité représente $8,5 \%$ du produit agricole national brut du pays (Ministère du Développement rural, 2001). Plus de
500000 personnes travaillent dans ce secteur. De la collecte des fruits à la production et la commercialisation du beurre, le karité est une activité essentiellement féminine (Elias et Carney, 2007). À partir des années 1990, un regain d'intérêt des décideurs politiques et des $\mathrm{ONG}$, au plan national et au plan international, a conduit à une multiplication des projets de développement du karité, en appui aux coopératives de femmes. Ces projets visent le marché international croissant des produits cosmétiques et pharmaceutiques (Elias et Saussey, 2013). La stratégie de soutien du gouvernement malien et des organismes de développement a consisté à privilégier le renforcement des capacités organisationnelles des femmes rurales, pour les aider à mieux se positionner sur les marchés d'exportation, présumés plus lucratifs. Cela devait leur permettre de s'approprier une part plus importante de la valeur ajoutée de la filière (Peppelenbos, 2008).

La COPROKAZAN (Coopérative des productrices de beurre de karité de Zantiébougou), autour de laquelle les activités de la PI ont été conduites, a été une pionnière. Son expérience lui a permis de saisir les opportunités de la filière du karité au Mali (Sidibé et al., 2012). Ses choix stratégiques par rapport à la collecte et à la transformation, ainsi que les liens qu'elle a maintenus avec les structures d'appui, sont présentés ci-dessous. Parmi ces structures d'appui figure le Sido (dont le nom signifie parc à karité en bambara), qui, malgré les difficultés rencontrées, reste la structure faîtière nationale des organisations féminines. Le Sido constitue une tentative de créer une structure interprofessionnelle afin de chercher des solutions aux problèmes auxquels la filière karité nationale est confrontée.

La COPROKAZAN a été créée en 1991 avec l'appui d'une ONG nationale, en vue de coordonner la collecte des amandes (les noix étant décortiquées par les productrices), leur transformation et la commercialisation du beurre de karité. Première coopérative du Mali ayant bénéficié d'appuis extérieurs, elle avait pour but de capitaliser les connaissances traditionnelles et le savoir-faire des femmes rurales autour du karité. Cela en vue de leur assurer des revenus auxquels elles ne pouvaient prétendre eu égard à leur accès limité aux opportunités supposées des marchés modernes. Comparée à d'autres coopératives, la COPROKAZAN a acquis une relative autonomie vis-à-vis des différentes organisations d'appui (Sidibé et al., 2012). Elle est ainsi devenue une source d'inspiration pour d'autres coopératives, nationales et étrangères.

Depuis sa création, la COPROKAZAN a évolué pour suivre le rythme des changements dans le secteur karité et son environnement (Sidibé et al., 2012). La forme organisationnelle actuelle de la COPROKAZAN est l'aboutissement de ces adaptations (Sidibé, 2013).

La plate-forme d'innovation a vu le jour début 2011, dans le cadre du programme de recherche-action Convergence des Sciences-Renforcement des systèmes d'Innovation - CoS-SIS (Röling et al., 2014). Elle était composée de dix membres, dont quatre issus de la COPROKAZAN et six venus des structures d'appui aux coopératives féminines de karité: l'Assemblée permanente des chambres d'agriculture du Mali (APCAM), la Chambre de commerce et d'industrie du Mali (CCIM), le ministère de la Promotion des femmes et de l'enfant, la recherche (l'Institut d'économie rurale - IER), et deux ONG. Les ONG étaient chargées du suivi des activités et des 
réunions de la plate-forme. La représentante du ministère était le point focal de la plate-forme et la responsable de la coordination et du partage d'information entre les membres. Elle était également chargée de l'organisation des rencontres avec les structures sollicitées pour appuyer les activités de la plate-forme.

La première réunion de la plate-forme a permis aux membres de réfléchir aux résultats de la phase exploratoire et du diagnostic conduits par le doctorant du domaine (Hounkonnou et al., 2012) ainsi qu'à leurs propres expériences. Ils ont ainsi livré une première analyse des conditions sociotechniques et institutionnelles entravant les performances des coopératives. L'analyse a consisté en une identification systématique des structures mais aussi des particularités et des intérêts, qui composent l'environnement institutionnel et le cadre réglementaire de la filière karité. Les membres ont échangé sur les changements à opérer en vue de lever les obstacles identifiés ainsi que sur les actions à tester par la plateforme et d'autres acteurs afin d'améliorer la filière. Ils ont examiné les acteurs dont les pratiques et les décisions devraient changer. Ces échanges ont permis d'identifier quatre priorités relatives à l'accès aux services et aux opportunités, qui entravent, selon eux, les performances locales et limitent le supposé potentiel international. Les priorités identifiées étaient :

- le fonds de roulement;

- la participation au Salon international de l'agriculture à Paris ;

- les équipements et le transport;

- l'emballage pour les produits issus du karité.

Les actions prioritaires consistaient à apporter des améliorations dans les dimensions suivantes: les attitudes, les procédures, les déficits d'information et les incitations à la performance matérielle (Avelino et Rotmans, 2009; Fuchs et Glaab, 2011). La mise en place d'un fonds de roulement était une priorité de longue date pour permettre à la coopérative d'explorer de nouvelles opportunités (Sidibé et al., 2012). À cet effet, la plate-forme a fait de l'établissement de partenariats entre la COPROKAZAN et les institutions financières une priorité stratégique.

L'institution de microfinance Soro Yiriwaso a été la première à prêter une oreille attentive aux sollicitations de la plate-forme. En effet, les institutions financières hésitaient jusque-là à investir dans ce secteur à cause du caractère fluctuant de la production de karité. C'est dans ce contexte que Soro Yiriwaso a octroyé un premier prêt à la coopérative, dont les implications sur le secteur seront analysées dans cet article.

\section{Objectifs et méthodes}

L'étude a couvert la période allant de 2011, début des activités de la plate-forme, à 2016. Elle visait à contribuer à l'analyse des performances collectives du secteur karité au Mali (Sidibé, 2013) et à fournir des informations utiles pour l'amélioration de la compétitivité de ce secteur. La méthodologie a consisté en une étude de cas autour des activités d'une plate-forme co-créée conjointement par une coopérative spécifique et d'autres acteurs du secteur dans le cadre d'un programme de recherche-action. Les activités de la plate- forme ont été considérées comme une expérience, afin d'identifier et d'analyser les processus sous-jacents qui ont conduit aux changements observés (Perri et Bellamy, 2012). L'accent a été mis sur une triangulation entre les interviews des acteurs du secteur, la documentation des performances de la plate-forme et les évènements significatifs de l'évolution du contexte. Cela a permis d'examiner comment un appui extérieur accordé à une coopérative catalyse les processus qui affectent les arrangements institutionnels et favorise l'innovation. La dynamique était analysée tous les quatre mois lors d'ateliers que le programme de recherche organisait avec les pairs des autres PI des trois pays impliqués dans le projet Cos-SIS (Benin, Ghana et Mali).

Des informations supplémentaires ont été collectées à travers des interviews semi-structurées. Ces 15 interviews ont été réalisées entre 2014 et 2016 avec des membres de la coopérative et d'autres acteurs de la filière karité, croisés souvent de façon inopinée. Une codification des réponses a permis de faire une analyse qualitative des notes prises lors de ces interviews (Tab. 1).

\section{Présentation et analyse des faits}

Cette section présente les quatre priorités sociotechniques et institutionnelles retenues et analyse la façon dont elles ont été traitées par la plate-forme.

\subsection{Facilitation de l'accès au fonds de roulement}

$\mathrm{Au}$ départ, la filière karité était fortement dépendante d'appuis extérieurs. Ces appuis étaient destinés au renforcement des capacités organisationnelles des coopératives et du leadership des femmes, et à des dotations en équipements et infrastructures. Dans certains cas, un fonds de roulement de départ était octroyé à la coopérative par les structures d'appui pour favoriser la production centralisée et maintenir un standard de qualité du beurre. En effet, la gestion de la qualité nécessite des prétraitements spécifiques des amandes achetées auprès des productrices individuelles. Une production centralisée dans les locaux de la coopérative avait été adoptée en réponse à l'hétérogénéité de la qualité des beurres de karité résultant des différentes techniques de production individuelles. La coopérative avait été confrontée à ce problème de qualité dès les expériences initiales de vente groupée de beurres produits individuellement (Tab. 2).

L'ONG AMPJ (Association malienne pour la promotion des jeunes) était le principal appui de la COPROKAZAN. Elle a facilité l'accès de la coopérative aux premiers équipements et infrastructures ainsi qu'au fonds de roulement initial. La mise en œuvre des choix stratégiques de la coopérative par rapport à l'achat des amandes et à la centralisation de la production de beurre était liée aux appuis de l'ONG. Forte de l'expérience de la première initiative de production centralisée, la coopérative a multiplié les sous-unités d'achat d'amandes et de production centralisée de beurre dans les villages. Cela afin d'élargir le réseau d'approvisionnement et le domaine d'influence de la coopérative à de nouvelles femmes, y compris des nonmembres; ces dernières pouvaient en effet vendre leurs amandes de qualité à la coopérative, à travers des membres. L'élargissement avait également pour but de stabiliser les 
Tableau 1. Description synthétique du contexte de l'étude avant la plate-forme d'innovation (source: Sidibé, 2013).

Table 1. Synthetic description of the study context before the innovation platform (source: Sidibé, 2013).

\begin{tabular}{ll}
\hline Année & Évènement \\
\hline 1991 & Appui à la création du groupement des femmes dans le village de Falaba par l'ONG AMPJ \\
$1992-1994$ & Appui à la construction d'infrastructures à Zantiébougou par l'ONG AMPJ \\
$1995-2000$ & Vente groupée du beurre de karité produit par les femmes individuelles \\
$2000-2004$ & Rejet par les acheteurs de Bamako d'importants volumes de beurre pour qualité non-uniforme \\
Mi-2004 & La coopérative COPROKAZAN est créée sur recommandation d'une étude diagnostique conduite par un consultant extérieur. \\
2005 & On distingue une minorité de femmes membres et une majorité de femmes non-membres. \\
2006 & Début de la gestion des stocks d'amandes pour la production centralisée de beurre de qualité uniforme au sein de la coopérative \\
Début 2010 SIDO est créé dans la perspective d'interprofession autour du karité. & Prêt bancaire (BNDA) de 3000 Euros de fonds de roulement à la coopérative \\
\hline
\end{tabular}

Tableau 2. Description synthétique du contexte de l'étude après la plate-forme d'innovation (source: notes de recherche). Table 2. Synthetic description of the study context after the innovation platform (source: field notes).

\begin{tabular}{ll}
\hline Année & Évènement \\
\hline 2011 & Création : La plate-forme d'innovation est créée autour de la coopérative avec l'appui du programme de recherche COS-SIS. \\
& Intermédiation : La plate-forme d'innovation a pour objectif d'apporter des innovations dans l'arrangement institutionnel \\
& autour du karité. \\
& Elle établit des liens entre la COPROKAZAN et les organismes de microfinance. \\
& L'intermédiation a pour but d'éliminer le conservatisme des organismes de finance et le particularisme basé sur l'idée de projet. \\
& Faciliter la mise en place de fonds de roulement pour améliorer les activités de la coopérative et créer de nouvelles \\
& opportunités. \\
& Un premier prêt de 23 000 Euros scelle le début du partenariat entre la coopérative et l'organisme de microfinance Soro Yiriwaso. \\
& Réplication : Le partenariat entre la coopérative et Soro Yiriwa se poursuit à travers d'autres prêts. \\
& Le partenariat entre la coopérative et Soro Yiriwa s'élargit à d'autres coopératives de femmes à travers le pays. \\
2012 & Les activités de la plate-forme d'innovation prennent fin. \\
& Diffusion : Soro Yiriwaso se positionne comme partenaire stratégique des coopératives de femmes du secteur karité. \\
\hline
\end{tabular}

approvisionnements en prenant en compte la fluctuation naturelle de la production de karité (Sidibé et al., 2014). Pendant ce processus, l'AMPJ a été confrontée à des problèmes de ressources financières ; la discontinuité du financement est alors devenue une menace majeure pour les activités de base de la coopérative. L'accès à un fonds de roulement suffisant est donc devenu le souci prioritaire de la coopérative.

Avant la création de la PI, les tentatives entreprises par la coopérative pour obtenir des prêts auprès des structures de financement, afin d'augmenter son fonds de roulement, n'avaient pas été fructueuses. Le déficit d'information et la prudence du personnel de ces structures de financement à s'engager dans de nouveaux projets n'étaient pas encourageants. Ce personnel hésitait à investir dans un domaine réservé aux femmes et basé sur un produit de cueillette dont la production reste aléatoire. Toutefois, la BNDA (Banque nationale de développement agricole) a octroyé un premier prêt; cependant, en plus d'un retard dans le décaissement, elle l'a plafonné tout en imposant un taux d'intérêt élevé et un échéancier de remboursement qui commençait, sans délai de grâce, le mois suivant le décaissement.

L'une des priorités de la PI était donc de faciliter des échanges ouverts entre la coopérative et les organismes financiers. L'inclusion des femmes de la coopérative dans la PI et la présence d'autres membres ont favorisé les progrès qu'elle a pu réaliser. Cependant, parmi les quatre structures de financement auprès desquelles une intermédiation a été menée, seule une organisation de microfinance dénommée Soro Yiriwaso a répondu favorablement.

Soro Yiriwaso a fait visiter les locaux de la coopérative par son personnel, pour évaluer ses capacités de remboursement, avant de signer un protocole d'accord pour un premier prêt de 15 millions de Francs CFA (23 000 Euros) en 2011, prêt assorti du dépôt des titres de propriété des infrastructures de la coopérative comme garantie. La signature de l'accord a été faite avec la PI comme témoin, engagée à jouer le rôle d'arbitre pouvant rapprocher les positions en cas de conflit entre les deux parties. Comme mesure d'accompagnement, la plateforme a pris des dispositions pour former les membres de l'équipe de gestion de la coopérative, de façon à s'assurer de l'utilisation efficiente du fonds de roulement. Après le remboursement réussi de ce premier prêt, l'expérience a été renouvelée deux années de suite et se poursuit aujourd'hui sans la facilitation de la plate-forme. Les membres de la coopérative expliquent avoir acquis, désormais, la capacité d'avoir des discussions directes avec l'organisation de microfinance. Ce qui est la preuve d'un apprentissage collectif réussi. Riche de cette expérience, la plate-forme a facilité l'accès de six autres coopératives confrontées aux mêmes défis, à des fonds de roulement empruntés auprès de Soro Yiriwaso. Ce qui a permis à Soro Yiriwaso de se positionner comme partenaire stratégique spécialisé dans un secteur qui jadis n'attirait pas les institutions de financement. 


\subsection{Participation au salon international de l'agriculture de Paris}

Nos travaux ont produit une analyse critique des aléas du marché international, marché qui conduit à négliger les potentialités et les opportunités du marché domestique (Sidibé et al., 2012). Ces études ont montré qu'un repositionnement sur les opportunités locales ainsi qu'un raffermissement des liens avec les commerçants locaux servaient mieux les intérêts des femmes que l'accès direct au marché international promu par les ONG et les agences de développement (Sidibé, 2013).

Cependant, avant ces travaux, la pensée dominante dans le secteur du karité tendait à faire de la participation des coopératives de femmes aux expositions internationales une opportunité de visibilité aux yeux des décideurs, d'accès à de nouveaux marchés et de développement de nouveaux partenariats. Les activités de la plate-forme étaient également guidées par cette pensée. Le choix de participer à des événements comme le Salon international de l'agriculture de Paris relève conjointement de la Chambre de commerce et de la Chambre d'agriculture. Les représentantes des femmes maliennes sont cependant choisies par le ministère de la Promotion des femmes et de l'enfant. Le ministère avait tendance à privilégier les coopératives appuyées par son partenaire du Programme des Nations unies pour le développement (PNUD). La circulation de l'information entre les structures était erratique. Le processus manquait de transparence et laissait peu de chance à d'autres coopératives de participer (Sidibé et al., 2012). Cette coordination peu équitable est illustrée par ce point de vue d'un acteur: "nous étions des acteurs du même domaine, mais nous nous détestions cordialement $»$.

La COPROKAZAN n'avait jamais été invitée à participer au salon de Paris. En 2011, un membre de la plate-forme, délégué et vice-président de la Chambre d'agriculture, était aussi membre du comité de gestion du programme CoS-SIS. Cela lui a conféré un rôle important d'intermédiaire entre la coopérative, la plate-forme et la Chambre d'agriculture. Quand le problème de la participation de la COPROKAZAN s'est posé lors de la réunion de la plate-forme, il s'est révélé être la personne en charge de ces questions. Séance tenante, il a appelé le président de la Chambre pour solliciter cette participation. La présidente de la coopérative, qui a conduit la délégation de la coopérative au salon de Paris de 2011, s'est félicitée à son retour des retombées de cette participation, qui a permis d'écouler tout le stock envoyé à Paris. Cependant, elle a regretté que les promesses de partenariat obtenues à Paris avec des clients potentiels n'aient pu être concrétisées.

\subsection{Dotation en charrettes et en équipements de transformation}

Les femmes collectent les fruits du karité dans les champs et les jachères, puis les transportent sur la tête vers leur domicile, sur une distance moyenne de $3 \mathrm{~km}$. Il avait été estimé lors des discussions au sein de la plate-forme qu'une dotation en charrettes aurait allégé la tâche des femmes et contribué à une augmentation du volume des noix collectées.

Le délégué de la Chambre d'agriculture a déclaré que la plate-forme pourrait compter sur le volet équipement du
Programme national d'appui à la productivité agricole (PAPAM) géré par la Chambre et destiné à faciliter l'accès des producteurs aux équipements, y compris les charrettes. Il avait été proposé de chercher un mode de gestion collective des charrettes par les groupes de femmes dans les villages. La plate-forme a travaillé sur ce mode de gestion collective et a formulé une requête d'équipements pour soumission à la Chambre d'agriculture. La requête a été élargie à d'autres équipements de collecte des fruits sur les conseils de la personne de contact du PAPAM, ce qui a augmenté le montant de la requête, qui a atteint 17,5 millions de FCFA (27000 Euros). Elle a été soumise le 15 avril 2012 mais est restée sans suite jusqu'à la fin des activités de la PI. Les démarches de la plate-forme sont donc restées vaines sur ce point.

\subsection{Les emballages pour le beurre de karité}

En dépit des risques que présente le marché international, l'importance que lui accordaient les acteurs de la filière karité faisait croire à beaucoup que les emballages étaient un facteur déterminant dans la compétitivité du beurre de karité du Mali. Lors des participations aux expositions internationales, les acteurs avaient comparé leurs emballages avec ceux des producteurs de karité d'autres pays, comme le Ghana. Les emballages et les contenants de la COPROKAZAN étaient initialement importés du Sénégal. À la rupture de ce canal d'approvisionnement (pour des raisons techniques), ils se sont tournés vers des fournisseurs ghanéens. Mais le Ghana étant un pays producteur de karité, il a été perçu comme un concurrent potentiel sur le marché international. Ils ont donc cherché des fournisseurs au Mali.

Les membres de la plate-forme ont alors engagé un processus d'intermédiation pour établir un partenariat entre les coopératives de femmes et les industries maliennes qui produisent les emballages et les contenants. Ces efforts d'intermédiation de la plate-forme n'ont cependant pas produit les résultats attendus. En effet, les discussions ont vite révélé que ce partenariat ne pourrait pas compenser les investissements nécessaires pour mettre à niveau les chaînes de production pour répondre aux exigences des coopératives en termes de type et de qualité des emballages. Par ailleurs, les coopératives ne semblaient pas constituer un marché suffisant pour les industries d'emballage locales. Finalement, les coopératives ont donc continué à importer les emballages du Ghana.

\section{Discussion}

Le rôle de la plate-forme dans l'identification des quatre actions prioritaires pour la filière a été déterminant. Cependant, seulement deux des quatre priorités fixées, l'accès au fonds de roulement et la participation au Salon international de l'agriculture de Paris, ont pu trouver des solutions. Nous allons analyser pourquoi la plate-forme a pu permettre de faire évoluer certaines conditions sociotechniques et institutionnelles et pas d'autres.

La plate-forme est perçue comme un espace social dans lequel des expériences d'innovations institutionnelles se conçoivent dans un contexte de rapport de forces et 
d'identification d'objectifs collectifs (Hounkonnou et al., 2016). Dans cette perception, la causalité du changement institutionnel est implicitement attribuée à la coordination et aux connaissances internes de la plate-forme. La discussion se propose de tester cette perception et de voir plus en profondeur si des processus sous-jacents peuvent être détectés pour expliquer le changement institutionnel ainsi que le rôle potentiel et les limites de la plate-forme d'innovation.

La première observation est que la focalisation des activités de la plate-forme sur les activités de base de la coopérative, l'achat des amandes, leur transformation et la vente du beurre de karité, a créé plus d'espace permettant à la coopérative de saisir de nouvelles opportunités auprès des organismes financiers. L'accès à un financement du fonds de roulement par une voie indépendante de celle de l'ONG, condition indispensable pour la conduite des activités de base de la coopérative, s'est trouvé être la plus facile à résoudre.

Quatre actions ont sans doute joué un rôle capital dans la résolution de ce problème :

- la garantie implicite symbolisée par la présence de la plateforme lors de la signature du protocole d'accord de prêt et l'offre d'arbitrage de celle-ci en cas de conflit;

- la visite des locaux de la coopérative par l'organisation de microfinance pour se convaincre sur le terrain des capacités de remboursement de son partenaire;

- l'utilisation des titres de propriété des infrastructures de la coopérative comme garantie physique;

- la formation en gestion de l'équipe de la coopérative pour une utilisation efficiente du fonds de roulement.

Cependant, le système ouvert (Pawson et Tilley, 1997) que constitue le secteur du karité laisse penser que les trois dernières actions auraient pu être impulsées par d'autres évènements ou d'autres interventions initiées indépendamment, par d'autres acteurs de développement actifs dans le secteur. De plus, l'effet de la participation dans l'équipe de négociation de quatre membres de la coopérative mérite d'être noté. Cela rend difficile la séparation des causes qui ont facilité la recherche de solution à cette priorité, notamment entre la part attribuable à l'expérience individuelle des membres de la coopérative et celle attribuable à la plate-forme les regroupant. Or les actions s'expliquent souvent par des causes multiples et solidaires. Le rôle catalytique qu'a pu jouer la plate-forme (ou qu'aurait pu jouer toute autre intervention extérieure), suggère que ce rôle soit perçu comme une contribution plutôt que seule cause de changement.

L'autre intérêt de cette action d'appui à la recherche de financement est la reproductibilité de l'expérience: au delà de l'accès de la COPROKAZAN à ces financements, d'autres coopératives ont ensuite pu aussi bénéficier de cette expérience.

La participation au Salon international de l'agriculture de Paris apparaît être le résultat de l'action d'une personne-clé plutôt que de celle de la PI en tant que collectif. Cette personne était certes membre de la plate-forme, mais la COPROKAZAN aurait-elle obtenu cette participation si elle ne l'avait pas été ? Certes, la plate-forme pourrait être créditée d'avoir ajouté des éléments persuasifs à la procédure officielle de prise de décision. Elle a sans doute permis à la coopérative d'être informée de l'existence de l'opportunité, et d'exprimer en face des «autorités» que représente la plate-forme, son sentiment d'avoir été injustement oubliée. Ce qui n'est cependant pas évident, c'est de savoir si la personne qui a joué le rôle principal avait ou non l'intention de prendre la même décision avant la réunion de la plate-forme, et indépendamment de sa participation à celle-ci.

Une autre limite de cette participation au Salon de Paris est sa non-reproductibilité : les différentes coopératives sont en compétition les unes avec les autres pour avoir accès à ce salon, à travers des contacts personnels avec les décideurs gouvernementaux. Il s'agit donc plus ici d'une expérience de résolution ponctuelle d'un problème que d'élaboration d'une solution diffusable à d'autres agents.

Pour ces deux actions, financement et Salon de Paris, nous soutenons que les membres de la plate-forme ont joué le rôle «d'entrepreneurs institutionnels» (van Paassen et al., 2014), en exerçant un mélange de compétences personnelles pour accéder aux instances de décision et à des réseaux extérieurs pour ouvrir la voie du changement. Cette observation incite à un appel, pour les membres des plates-formes engagés dans un processus d'innovation, à se focaliser plus sur la façon dont les choses s'accomplissent dans la pratique et moins sur leur rôle de représentants des enjeux et des intérêts particuliers des organisations dont ils sont issus (Klerkx et Aarts, 2013). Tout en reconnaissant l'importance du choix stratégique des membres de la PI comme l'une des conditions de succès, nous estimons que le concept de «membre-clé » attribue l'effet à un individu plutôt qu'à l'action collective de la plate-forme. En mettant l'accent sur l'action collective, nous dépersonnalisons le travail institutionnel pour porter la réflexion sur ce qui peut se passer lorsqu'une coopérative est aidée de façon proactive par un acteur extérieur pour influencer et co-créer une PI.

L'hypothèse du programme CoS-SIS qui stipule que les conditions sociotechniques (l'achat d'amandes avec les femmes individuelles et la production centralisée de beurre de karité par une coopérative) et institutionnelles (accès au fonds de roulement) ouvrent la voie à l'innovation, est confirmée. Cependant, nous estimons que les résultats découlent de l'effet combiné d'évènements et de processus contextuels en interaction avec la dynamique du système ouvert.

En effet, le fonds de roulement a catalysé un processus déjà en cours dans la coopérative : celui de la centralisation de la production de beurre et de la multiplication des sousunités de production centralisée dans les villages. Ce qui aurait pu être catalysé par d'autres appuis extérieurs. Ainsi, les processus par lesquels une intervention extérieure comme les plates-formes d'innovation produisent des résultats, dépendent de la façon dont elles s'alignent sur les pratiques des acteurs de base, comme l'approvisionnement de la coopérative en amandes et la production de beurre de qualité homogène. Cette articulation entre l'intervention extérieure et les pratiques en cours permet de créer des conditions favorables à la saisie des opportunités qui se présentent aux acteurs.

Les deux priorités n'ayant pas trouvé de solutions montrent les limites de la composition actuelle de la plate-forme. Il est difficile de motiver des acteurs non liés par des valeurs professionnelles antérieures, à jouer leur rôle potentiel dans un secteur donné. C'est le cas des industries d'emballage, qui n'avaient pas une expérience de collaboration antérieure avec les coopératives de karité. Ce point pourrait soulever des 
questions liées à la gestion de l'information et à la façon dont la connaissance est produite entre acteurs du même secteur. Dans les deux cas, les charrettes et les emballages, les acteurs dont l'assistance a été sollicitée n'avaient pas d'informations préalables sur le secteur, et encore moins sur la COPROKAZAN. Ils n'ont pas été invités à une coproduction de la connaissance et à un apprentissage mutuel à travers une expérimentation conjointe avec la coopérative et la plateforme. Cela nous amène à penser que l'innovation effective a des chances de se réaliser lorsque l'interaction est orientée vers la résolution de problèmes qui affectent des acteurs qui partagent les mêmes valeurs professionnelles. Les soucis liés aux problèmes de mise en place du fonds de roulement et de méfiance des organismes de financement pour le secteur karité constituent des valeurs que partagent les coopératives et les organismes financiers. L'innovation est visible à travers l'élargissement du partenariat avec Soro Yiriwaso à d'autres coopératives de femmes à travers le pays. Ce qui fait aujourd'hui de Soro Yiriwaso le premier partenaire stratégique des coopératives de femmes du secteur du karité au Mali. Les transactions commerciales directes, comme celle autour des emballages, et les procédures guidées par l'idée de projet, comme celles relatives à l'acquisition de charrettes et d'équipements à travers le programme PAPAM de la Chambre d'agriculture, pourraient avoir peu de chance de produire de l'innovation institutionnelle dans le secteur du karité. Elles sont en effet moins régies par des valeurs partagées que les acteurs aimeraient perpétuer.

Le partenariat développé entre la COPROKAZAN et l'organisation de microfinance Soro Yiriwaso a permis à celleci de découvrir une nouvelle opportunité d'affaire, dont l'octroi ultérieur de fonds de roulement à six autres coopératives de femmes est une illustration. Cette collaboration avec les coopératives des femmes, qui se poursuit actuellement, a permis à Soro Yiriwaso de se positionner comme partenaire stratégique du secteur du karité. Le rôle joué par la plate-forme d'innovation dans ce cas indique qu'elle a catalysé l'émergence de proto-institutions (Lawrence et al., 2002), ou de précurseurs d'institution dotés du potentiel de faire partie des mécanismes de l'ensemble de la filière.

La non-pérennisation de la plate-forme après le programme de recherche qui l'a suscitée, ainsi que son incapacité à résoudre certains problèmes, indiquent que la plate-forme pourrait être considérée comme une mesure temporaire visant à apporter des solutions à des problèmes spécifiques, définis dans le temps et dans l'espace (Vellema et al., 2011).

\section{Conclusion}

Cet article a analysé quatre initiatives prises par une plateforme d'innovation afin d'apporter des changements bénéfiques à une coopérative et à la filière karité du Mali dans son ensemble. Deux ont été couronnées de succès et deux autres ne l'ont pas été. Nous en concluons que l'innovation a des chances de se réaliser lorsque le processus est inspiré par la recherche de solutions à des problèmes concrets, destinées à appuyer les acteurs ayant des enjeux directs et des valeurs professionnelles partagées dans le domaine visé.

La plate-forme pourrait être considérée comme un outil temporaire de recherche de solutions à des problèmes spécifiques plutôt qu'un projet d'intervention à pérenniser. Les changements impulsés par la plate-forme peuvent avoir le potentiel de devenir de nouvelles institutions pour le secteur. Cependant, il convient de ne pas attribuer de façon simpliste et normative la causalité des changements observés aux seules activités de la PI. Il y a une distinction à faire entre les résultats attribuables à un individu clé et ceux attribuables collectivement à une plate-forme. Nous pensons également qu'il est difficile de développer des actions d'expérimentation conjointe avec des acteurs n'ayant aucun lien antérieur avec un domaine. L'expérimentation conjointe autour du prêt financier était, $a$ priori, destinée à favoriser la reconnaissance par les parties de leurs intérêts et valeurs professionnelles mutuelles. Elle visait moins à défendre les intérêts de ceux ayant déjà un enjeu connu et déterminés à développer de nouvelles perspectives pour leurs pratiques: concurrencer l'emballage extérieur par exemple. Les plates-formes ne constituent donc pas des baguettes magiques pouvant toujours garantir des résultats positifs. La clé du succès est pour beaucoup dans la façon dont les activités de la plate-forme parviennent à entrer en résonnance avec les autres expériences locales en cours.

Remerciements. Les auteurs remercient Janice Jiggins pour le temps et les efforts qu'elle a consacrés à la lecture critique de cet article. Nous remercions également les évaluateurs anonymes pour les critiques constructives et les suggestions qui ont permis d'améliorer le manuscrit.

\section{Références}

APROMA. 1995. Étude de la filière karité du Burkina Faso. Rapport principal. Contrat $n^{\circ}$ 01/DEL/94. Bamako, Mali: Association des produits à marché, Vol. 168, p. + annexes p.

Avelino F, Rotmans J. 2009. Power in transition: an interdisciplinary framework to study power in relation to structural change. European Journal of Social Theory 12(4): 543-569.

Derks E, Lusby F. 2006. Mali Shea Kernel: value chain case study. ACDI/VOCA. Accelerated microenterprise advancement project (AMAP)

Elias M, Carney J. 2007. African shea butter: a feminized subsidy from nature. Africa-london-international african institute 77(1): 37.

Elias M, Saussey M. 2013. 'The Gift that keeps on giving': unveiling the paradoxes of fair trade shea butter. Sociologia Ruralis 53(2): 158-179.

Fold N, Reenberg A. 1999. In the shadow of the 'chocolate war': local marketing of shea nut products around Tenkodogo, Burkina Faso. Geografisk Tidsskrift / Danish Journal of Geography Special Issue 2: 113-123.

Fuchs D, Glaab K. 2011. Material power and normative conflict in global and local agrifood governance: The lessons of 'Golden Rice'in India. Food Policy 36(6): 729-735.

Greig D. 2006. Shea butter: connecting rural Burkinabè women to international markets through fair trade. Development in Practice 16(5): 465-475.

Henry A, Chithra V, Nair N. 1983. Vitellaria vs. Butyrospermum (Sapotaceae). Taxon 32(2): 282-286.

Hounkonnou D, Kossou D, Kuyper TW, Leeuwis C, Nederlof ES, Röling N, et al. 2012. An innovation systems approach to institutional change: smallholder development in West Africa. Agricultural Systems 108: 74-83.

Hounkonnou D, Brouwers J, van Huis A, Jigginss J, Kossou D, Röling N, et al. 2016. Triggering regime change: a comparative 
analysis of the performance of innovation platforms that attempted to change the institutional context for nine agricultural domains in West Africa. Agricultural Systems 108(2012): 74-83.

Klerkx L, Aarts N. 2013. The interaction of multiple champions in orchestrating innovation networks: conflicts and complementarities. Technovation 33(6): 193-210.

Klerkx L, Aarts N, Leeuwis C. 2010. Adaptive management in agricultural innovation systems: the interactions between innovation networks and their environment. Agricultural Systems 103(6): 390-400.

Lawrence TB, Hardy C, Phillips N. 2002. Institutional effects of interorganizational collaboration: the emergence of proto-institutions. Academy of Management Journal 45(1): 281-290. Doi $10.2307 / 3069297$.

Leeuwis C. 2004. Changing views of agricultural innovation: implications for communicative intervention and science. Research to impact: case studies for natural resource management for irrigated rice in Asia 2010: 15-32

Lusby F, Derks E. 2006. Shea kernels from Mali: a value chain case study. Small enterprise development 17(2): 36-46.

Ministère du Développment rural. 2001. Schéma Directeur du secteur du développement rural (SDDR). Bamako: Ministère du Développment rural - Cellule de planification et de statistique.

Nederlof ES, Pyburn R. 2012. One finger cannot lift a rock: facilitating innovation platforms to trigger institutional change in West Africa. Amsterdam: KIT Publishers, $144 \mathrm{p}$.

Nederlof S, Wongtschowski M, van der Lee F, eds. 2011. Putting heads together: agricultural innovation platforms in practice. Amsterdam: KIT Publishers, KIT Development, Policy \& Practice.

Pawson R, Tilley N. 1997. Realistic evaluation. London: Sage, 235 p.

Peppelenbos L. 2008. Pro-poor market development: an approach and quick-scan screening tool for pro-poor business propositions. KIT working papers series C1. Amsterdam: KIT.
Perri 6, Bellamy C. 2012. Principle of Metodology: research design in social science. London, UK: Sage, 324 p.

Röling N, Jiggins J, Hounkonnou D, van Huis A. 2014. Agricultural research - from recommendation domains to arenas for interaction: experiences from West Africa. Outlook on Agriculture 43(3): 179-185. DOI: 10.5367/oa.2014.0172.

Sidibé A. 2013. Analysis of collective performance in the Malian shea sector: from fields to markets. Netherlands: Wageningen University, $124 \mathrm{p}$

Sidibé A, Vellema S, Dembélé F, Traoré M, Kuyper TW. 2012. Innovation processes navigated by women groups in the Malian shea sector: How targeting of international niche markets results in fragmentation and obstructs co-ordination. Njas-Wageningen Journal of Life Sciences 60-63: 29-36. DOI: 10.1016/ j.njas.2012.06.010.

Sidibé A, Vellema S, Dembélé F, Témé B, Yossi H, Traoré M, et al. 2014. Women, shea, and finance: how institutional practices in a Malian cooperative create development impact. International Journal of Agricultural Sustainability 1-13. DOI: 10.1080/ 14735903.2014.909640.

Spielman DJ. 2005. Innovation systems perspectives on developingcountry agriculture: a critical review. International food policy research institute (IFPRI). International service for national agricultural research (ISNAR) division.

van Paassen A, Klerkx L, Adu-Acheampong R, Adjei-Nsiah S, Zannoue E. 2014. Agricultural innovation platforms in West Africa: how does strategic institutional entrepreneurship unfold in different value chain contexts? Outlook on Agriculture 43(3): 193-200.

Vellema S, Nakimbugwe D, Mwesige D. 2011. The Ugandan oil seed sub-sector platform. In: Nederlof S, Wongtschowski M, Lee FVD, eds. Putting heads together: agricultural innovation platforms in practice. Amsterdam: KIT publishers, pp. 114-123.

Citation de l'article : Sidibé A, Vellema S, Dembelé F, Traoré M, Kuyper TW. 2017. Analyse d'une plate-forme d'innovation dans la filière karité au Mali. Cah. Agric. 26: 45001. 